\title{
Answer to the Letter to the Editor of H. Sarman et al. concerning "The prevalence of depressive symptoms before and after surgery and its association with disability in patients undergoing lumbar spinal fusion" by M. Wahlman et al.; Eur Spine J (2014) 23:129-134
}

\section{Wahlman}

Received: 30 November 2014/Revised: 30 November 2014/ Accepted: 30 November 2014/Published online: 3 January 2015

(c) Springer-Verlag Berlin Heidelberg 2014

Thank you very much for the interest in our study.

In our study we reported how the decrease of pain and improvement of disability after the surgery decreased the depressive symptoms in patients with chronic back pain. The change in depressive symptoms also correlated to the decrease of pain and improvement of disability, therefore, we believe that in these patients the main causes of the depression are pain and disability and by treating these we also may relieve depressive symptoms. However, in patients in who the depression is primary problem these antidepressants may work and we encourage for further studies evaluating also the influence of antidepressants.

Conflict of interest None.

M. Wahlman ( $\square)$

Department of Orthopaedic and Trauma Surgery,

Tampere University Hospital, Tampere, Finland

e-mail: marko.wahlman@fimnet.fi 